\title{
IMPLEMENTAÇÃO DE MUDANÇAS EM CURSO DE GRADUAÇÃO A DISTÂNCIA DA UFRGS E SUA AVALIAÇÃO PELOS ALUNOS
}

\author{
Mário César Carvalho - Fac. de Castanhal-PA - carvalhomario@ yahoo.com.br \\ Camila Furlan da Costa - UNIPAMPA - camilacfcosta@gmail.com \\ Marisa Rhoden - EA/UFRGS - misrhoden@ea.ufrgs.br \\ Luis RoqueKlering - PPGA/UFRGS e EGC/UFSC - 1rklering@via-rs.net
}

\begin{abstract}
Resumo
O presente texto constitui um relato analítico da implementação do curso de graduação em Administração na modalidade a distância, desenvolvido no período de 2006 até 2010, como projeto-piloto e parceria entre o Ministério da Educação (MEC), o Banco do Brasil (BB) e a Escola de Administração (EA) da Universidade Federal do Rio Grande do Sul (UFRGS). O curso iniciou com cerca de 600 alunos - advindos da denominada demanda interna (DI), ou seja, funcionários do BB, e da demanda social (DS), público em geral - e terminou com, aproximadamente, 350 alunos. Para seu desenvolvimento, utilizou a Plataforma NAVi como Ambiente Virtual de Aprendizagem. Na gestão do curso, com o passar do tempo, foram feitas mudanças na sua estrutura administrativa e pedagógica, com a introdução de novos papeis, decorrentes de adaptações às necessidades dos alunos e em prol de uma maior eficácia do processo de ensino e aprendizagem. O texto analisa como estas mudanças afetaram positivamente os índices de satisfação dos alunos do curso de graduação em Administração da EA/UFRGS, conforme pesquisas de avaliação das disciplinas cursadas.
\end{abstract}

Palavras-Chaves: novos papéis, avaliação de curso, Administração a distância, Educação a Distância.

\section{IMPLEMENTATION OF CHANGES IN UNDERGRADUATE COURSE IN DISTANCE MODE AND ITS EVALUATION BY STUDENTS}

\begin{abstract}
The present paper consists of an analytical report on the implementation of the undergraduate program in Administration in the distance learning modality, developed in the period from 2006 to 2010 as a pilot-project, by a partnership between the Ministry of Education (MEC), Banco do Brasil (BB) and the Administration School (EA) at the Federal University of Rio Grande do Sul (UFRGS). The course started with around 600 students from the so-called internal demand (ID), i.e., BB employees, and from the social demand (SD), the general public. It was completed with approximately 350 students. For its development, the NAVi Platform was used as the Virtual Learning Environment. During the course management, changes were introduced to its administrative and pedagogical framework, with introduction of new roles resulting from adaptations to the needs of students and aiming at greater effectiveness of the educational and learning process. The paper analyzes how these changes in the course framework and pedagogy positively affected the satisfaction rates of students in the EA/UFRGS undergraduate program in Administration, according to surveys evaluating the courses taken.
\end{abstract}

Keywords: new roles, course evaluation, Distance Learning Administration, Distance Learning. 


\section{INTRODUÇÃO}

O curso de graduação em Administração, objeto de análise deste texto, foi desenvolvido no período de 2006 a 2010, como projeto-piloto e parceria entre o Ministério da Educação (MEC), o Banco do Brasil (BB) e a Escola de Administração da Universidade Federal do Rio Grande do Sul (EA/UFRGS). O projeto iniciou no segundo semestre de 2006 com cerca de 600 alunos - advindos da denominada demanda interna (DI), ou seja, funcionários do BB, e da demanda social (DS), público em geral. Encerrou em 2010 com, aproximadamente, 350 alunos, quando já se encontrava dentro do sistema Universidade Aberta do Brasil (UAB).

O curso de graduação utilizou, para apoiar as suas atividades de ensino, a Plataforma NAVi, desenvolvida pelo Núcleo de Aprendizagem Virtual (NAVi) da EA/UFRGS (KLERING e SCHRÖEDER, 2011). Tanto a estrutura tecnológica, quanto as estruturas administrativa e pedagógica do curso de graduação passaram por mudanças desde seu início, em 2006. Atenção especial neste texto é dada para a reflexão sobre o uso de recursos do Ambiente Virtual de Aprendizagem (AVA) NAVi e para a introdução de novos papéis decorrentes de adaptações às necessidades dos alunos e em prol da eficácia do processo de ensino e aprendizagem.

O presente texto, na forma de relato, visa, assim, a contribuir para a compreensão de como estas mudanças resultaram em aumento nos índices de satisfação dos alunos do curso de graduação em Administração da EA/UFRGS, conforme expresso por eles nos formulários de avaliação das disciplinas.

\section{O CURSO DE GRADUAÇÃO EM ADMINISTRAÇÃO A DISTÂNCIA DA EA/UFRGS}

A EA/UFRGS, localizada em Porto Alegre (RS), tem papel ativo e importante na implantação e disseminação de cursos na modalidade a distância, pois oferece cursos de capacitação a gestores de órgãos públicos; cursos de especialização de pequeno a grande portes; cursos de extensão em diferentes áreas e com diferentes portes; curso de graduação (no período de 2006 a 2010). Constitui também a Unidade Acadêmica que sediou o desenvolvimento inicial da Plataforma NAVi, utilizada como opção oficial de AVA da UFRGS.

Quase sem exceção, os cursos da EA (presenciais ou a distância) são desenvolvidos utilizando a plataforma NAVi, que possui entre seus principais recursos de aprendizagem: apresentação de informações gerais das disciplinas; notícias; vídeoaulas; conteúdo de aulas; agenda; fórum; acervo de informações; enquetes; aulas interativas (chats); exercícios; avaliações; portfólio; estudos de caso; relatos; suporte técnico; café virtual; correio interno; estatísticas de acesso; e scorm (KLERING e SCHRÖEDER, 2011).

Neste texto, opta-se por não detalhar todos os cursos e recursos disponíveis na EA/UFRGS; para fins de estudo, escolhe-se o curso de graduação em Administração a distância, projeto com maior duração - nove semestres - concluído em 2010. Este curso contribuiu para a implementação da política de ampliação do acesso ao ensino superior, bem como para o atendimento da demanda de qualificação de funcionários do BB.

A experiência anterior no desenvolvimento de cursos na modalidade a distância foi um dos critérios da escolha da UFRGS pelo MEC para sediar o oferecimento do curso de graduação em Administração a distância no Rio Grande do Sul (RS). O curso foi desenvolvido em módulos semestrais, totalizando 3.000 horas, executadas em 09 semestres e um total de 200 créditos. 
Feita a apresentação do curso objeto deste estudo, passa-se a descrever as principais mudanças ocorridas em sua estrutura desde seu início em 2006.

\section{MUdANÇAS NAS PRÁtiCAS ADMINISTRATIVAS E PEDAgÓgICAS E A INTRODUÇÃO DE NOVOS PAPEIS}

Apresentam-se nesta seção as mudanças e adaptações de práticas administrativas e pedagógicas realizadas no curso de graduação em Administração a distância da EA/UFRGS, que podem ter contribuído para o aumento dos índices de satisfação dos alunos no decorrer do mesmo. Inicialmente, descrevem-se as ações pedagógicas adotadas por meio da utilização do AVA NAVi, com destaque para os chats e fóruns. Em seguida, apresentam-se os papeis que foram introduzidos no curso.

\subsection{A UTILIZAÇÃO DOS RECURSOS DA PLATAFORMA NAVi}

O curso de graduação utilizava, nos seus três primeiros semestres (2006-2, 20071 e 2007-2), basicamente três funcionalidades da plataforma para condução das disciplinas: chat, fórum e portfólio. Os chats são os momentos de interação síncrona, que se assemelham a uma aula presencial. Todos têm direito de falar ao mesmo tempo e um tutor controla o ritmo das postagens, atuando como mediador em tempo real.

O fórum é um recurso para interação assíncrona onde alunos e tutores debatem um determinado tema em tempos distintos e de forma mais intercalada. Os fóruns são normalmente organizados por tópicos, com determinado prazo de duração. No curso em foco, tinham um prazo de uma semana. Após este período eram abertos novos tópicos e os alunos podiam acompanhar as instruções dos tutores em direção aos temas seguintes.

O portfólio é o local onde os alunos postam os arquivos editados fora da plataforma, utilizando softwares de edição de textos, planilhas e apresentações. Constitui apenas um destino (lugar) final para disponibilização (depósito) dos trabalhos acadêmicos, onde os tutores podem fornecer feedback individualizado sobre a produção intelectual do aluno.

Os planos de ensino das disciplinas apresentavam, até o ano de 2007, características que limitavam, em alguma medida, o processo de aprendizagem dos alunos. A partir do ano de 2008, algumas ferramentas da plataforma, como prova online, enquetes, relatos e estudos de caso, passaram a ser incluídas mais frequentemente na condução das disciplinas.

Esta diversificação no uso dos recursos trouxe mais variedade e dinamicidade às aulas. Em disciplinas que continham enfoques mais quantitativos, os tutores utilizavam enquetes e provas on-line durante os chats com o propósito de fazer os alunos exercitarem e verificarem seus conhecimentos em tempo real acerca dos conteúdos trabalhados.

Já em disciplinas com abordagem mais qualitativa, as ferramentas de relatos e estudos de caso eram mais apropriadas para cumprir os propósitos de aprendizagem e variação das dinâmicas.

No entanto, as modificações mais significativas no modo de utilização de recursos do AVA NAVi foram aquelas referentes à maneira de conceber e executar as atividades relacionadas a chats e fóruns. Estas alterações serão exploradas a seguir.

\subsubsection{Chats}

Uma característica marcante do curso de graduação da EA/UFRGS foi o acompanhamento dos alunos pelos tutores. Os chats, como principal forma de interação, 
caracterizavam esta proximidade. Aconteciam dois encontros semanais, em tempo real, com cerca de duas horas de duração cada.

A partir de março de 2008, passou a ser obrigatória a condução de chats em um dos laboratórios da EA/UFRGS. Antes, tutores e professores trabalhavam em ambientes diferentes (geralmente nas suas residências), em ritmos diferentes, com conhecimentos diferentes, e capacidades diferentes da internet de cada um. Isso fazia com que os tutores não cooperassem entre si, tampouco contassem com a ajuda dos professores para dirimir suas dúvidas em tempo real.

Nos primeiros meses dessa obrigação de trabalhar juntos, alguns tutores e professores se mostraram resistentes. Porém, os feedbacks das trocas de opiniões a respeito e das avaliações dos alunos foram determinantes para convencer que esta prática era benéfica para todos. Outrossim, o envolvimento dos docentes passou a ser maior, sendo que alguns inovaram ao entrar ao vivo nas salas de aula virtuais.

Os alunos gostaram da possibilidade de interagir ao vivo com os professores responsáveis pelas disciplinas. Já os tutores se sentiram intrigados com a presença de outra pessoa da equipe em sua sala virtual. Com o tempo, compreenderam o espírito de colaboração que se deve ter em EAD e até solicitavam a presença dos professores na interação com os alunos, quando não conseguiam responder a determinados questionamentos.

\subsubsection{Fóruns}

$\mathrm{Na}$ execução dos planos de ensino, verificou-se que os fóruns temáticos e fóruns de dúvidas não estavam sendo assimilados de maneira correta pelos alunos. Por natureza, o fórum deve ser um espaço de debates, esclarecimento de dúvidas e registro de participação dos alunos para fins de avaliação.

No entanto, a maior parte dos alunos do curso estava preocupada apenas com a quantidade e não com a qualidade das postagens. Por mais que se pensasse em questões de fóruns que efetivamente fossem geradoras de debates qualificados, as respostas dos alunos ainda traziam viés de obrigação, de quantidade de postagens para fins de avaliação.

Diante deste quadro, decidiu-se utilizar os fóruns somente para dirimir dúvidas de conteúdo e como apoio às demais tarefas previstas nos planos de ensino. A intenção foi qualificar a participação dos alunos neste recurso e ao mesmo tempo fazer com que os seus esforços fossem direcionados a atividades que exigiam empenho pessoal de realização, e que facilitariam o processo de avaliação.

\subsection{PAPÉIS}

A EA/UFRGS não possui um setor de EAD institucionalizado e estruturado. Cada projeto constitui sua própria equipe de gestão e de acompanhamento pedagógico. Até o ano de 2007, o curso de graduação contava com quatro figuras permanentes: uma professora coordenadora, dois técnicos administrativos - um como gerente e outro como responsável pelas planilhas de avaliação - e uma bolsista.

O padrão de estruturação das disciplinas era o seguinte: um professor era convidado para coordenar uma disciplina de 60, 90 ou 120 horas-aula; ele confeccionava sozinho ou em conjunto com outro(s) docente(s) o plano de ensino; outrossim, ou ele adotava a apostila nacional do projeto, ou fazia uma nova apostila, ou indicava referências bibliográficas para a disciplina; ele coordenava a equipe de colegas professores da disciplina; formava e geria a equipe de tutores; e fazia o acompanhamento das aulas. 
Com o passar do tempo, outros papeis foram sendo introduzidos na estrutura do curso e, por conseguinte, das disciplinas. Destaca-se nesse texto a assunção de três atores importantes no processo de ensino-aprendizagem: o coordenador de tutoria, o assistente de avaliação e os tutores-facilitadores.

\subsubsection{Coordenador de tutoria}

A partir do semestre de 2008-1, foi introduzida a figura do coordenador de tutoria no curso da graduação em foco, com o intuito de intensificar o acompanhamento dos chats, bem como servir de interlocutor com os vários atores (profissionais) que passavam pelo curso. Após alguns meses, a atuação deste coordenador se tornou indispensável para a uniformização e eficácia da implementação dos planos de ensino.

Desde a fase de planejamento da disciplina, a opinião e experiência trazidas por ele às reuniões entre coordenação, gerência, professores e tutores eram de grande valia para adequar e implementar as estratégias pedagógicas pensadas pelos responsáveis pelas disciplinas à realidade dos alunos e do contexto.

Com o reconhecimento deste profissional como sendo fundamental para a gestão do curso, ocorreram significativas melhoras nos índices de avaliação de quase todos os envolvidos nas rotinas do curso em foco.

O coordenador de tutoria passou a ser responsável por todo o processo de aperfeiçoamento da capacitação de tutores. Antes, tal capacitação era restrita a aspectos estritamente tecnológicos ligados ao uso da plataforma NAVi. A partir do ano de 2008, a capacitação de tutores e professores passou a ser ministrada por este profissional, que inseriu na programação do treinamento o debate: a) de questões pedagógicas peculiares à EAD; b) das competências dos tutores para atuar nesta modalidade de ensino; e c) de aspectos concernentes à avaliação em ensino superior.

Além disso, o acompanhamento pedagógico passou a ser diferenciado. Foi implementado um sistema que passou a permitir o acompanhamento em tempo real do lançamento das notas dos alunos em todas as disciplinas, tarefa por tarefa. Isso significou um grande avanço, pois a partir desta iniciativa era possível verificar todo o histórico de um aluno, sem a necessidade de recorrer-se a consultas formais em outras instâncias da EA. Tutores, professores, coordenadores e gerentes podiam acessar todas estas informações instantaneamente.

Com a criação deste e de outros mecanismos de acompanhamento pedagógico, houve a necessidade de se instituir um novo papel: o assistente de avaliação.

\subsubsection{Assistente de avaliação}

O assistente de avaliação, com a supervisão de um servidor da Universidade, foi um papel introduzido com a responsabilidade de proceder à abertura, correções e fechamentos de todas as planilhas de notas dos alunos. Além disso, de cuidar da divulgação das notas e dos requerimentos de revisão de conceitos.

Até o ano de 2007, este papel era desempenhado pelo bolsista de apoio administrativo à gerência. A função era, até então, mais operacional, ligada ao recebimento das planilhas enviadas por correio eletrônico e respectiva conferência. Como as tarefas foram ficando cada vez mais analíticas, percebeu-se a necessidade de destacar esta função, em relação ao papel anterior meramente operacional. A partir de 2008, o intitulado assistente de avaliação passou a trabalhar de maneira integrada com o coordenador de tutoria. 


\subsubsection{Tutor-facilitador}

O tutor-facilitador também foi outro papel introduzido a partir do semestre de 2008-1 no curso em foco. Este profissional passou a ter a função de auxiliar os professores no planejamento das disciplinas, principalmente no seu desenvolvimento operacional.

Passou a constituir uma função desempenhada normalmente por um tutor experiente em EAD e com domínio do conteúdo de uma disciplina. Realiza a interface entre o professor responsável por disciplina e a coordenação de tutoria, e é encarregado de gerenciar as informações entre a equipe de tutores, ou seja, é responsável pela padronização das condutas e pelo cumprimento dos prazos estabelecidos no plano de ensino.

A instituição deste papel contribuiu para elevar o índice de satisfação em relação ao planejamento das disciplinas e ao trabalho dos tutores.

\section{O IMPACTO DAS MUDANÇAS NA AVALIAÇÃO DAS DISCIPLINAS}

Após a apresentação do histórico do curso em foco, bem como das mudanças que ocorreram na estruturação do curso com a introdução de novos papeis, apresentamse os resultados das avaliações das disciplinas pelos alunos, antes e depois da introdução destes novos papeis, buscando-se ilustrar de forma quantitativa qual $o$ impacto destas mudanças.

Nos primeiros anos do curso (entre 2006-1 e 2008-1), os índices de aprovação das disciplinas oscilavam bastante. Isso acontecia porque as ações de planejamento das disciplinas ocorriam de forma desconectada, e dependiam essencialmente do esforço individual de professores e tutores em determinadas disciplinas. Pode-se observar isto na tabela 1 seguinte, em disciplinas como Economia, Psicologia e Matemática Financeira.

Tabela 1 - índices de aprovação dos professores e do planejamento de disciplinas

\begin{tabular}{|c|c|c|c|}
\hline \multicolumn{2}{|l|}{ Disciplinas 2006-2 } & \multicolumn{2}{|l|}{ Disciplinas 2007-1 } \\
\hline EAD & $78 \%$ & OSM & $75 \%$ \\
\hline TGA & $68 \%$ & Processo Decisório & $78 \%$ \\
\hline Sociologia & $72 \%$ & Matemática & $67 \%$ \\
\hline Ciência Política & $61 \%$ & Filosofia & $78 \%$ \\
\hline \multicolumn{2}{|l|}{ Disciplinas 2007-2 } & \multicolumn{2}{|l|}{ Disciplinas 2008-1 } \\
\hline Direito Administrativo & $59 \%$ & Sist. De informação & $60 \%$ \\
\hline Contabilidade & $49 \%$ & Matemática Financeira & $87 \%$ \\
\hline Economia & $82 \%$ & Administração Pública & $42 \%$ \\
\hline \multirow[t]{2}{*}{ Psicologia } & $86 \%$ & Estatística & $43 \%$ \\
\hline & & Antropologia & $96 \%$ \\
\hline \multicolumn{2}{|l|}{ Disciplinas 2008-2 } & \multicolumn{2}{|l|}{ Disciplinas 2009-1 } \\
\hline Resp. social corporativa & $89 \%$ & Gestão de pessoas & $84 \%$ \\
\hline Adm. Fin. e orçamentária & $92 \%$ & Marketing & $88 \%$ \\
\hline Economia Micro e macro & $79 \%$ & Gestão Ambiental & $91 \%$ \\
\hline Planejamento & $76 \%$ & Comércio exterior* & $88 \%$ \\
\hline
\end{tabular}

Fonte: base de dados de avaliação das disciplinas do curso de graduação. 
As ações integradas entre coordenação de tutoria, tutores-facilitadores e professores passaram a se mostrar de forma mais evidente e conectadas a partir da disciplina de Antropologia, ministrada no final do semestre 2008-1. Esta disciplina foi um divisor de águas na forma de construir e conduzir o plano de ensino. A equipe responsável pela disciplina trabalhou com quatro meses de antecedência na preparação de chats, fóruns e exercícios, fazendo com que todos tivessem domínio do conteúdo e das dinâmicas aplicadas. Além disso, a professora teve a atitude inédita de entrar nos chats em todas as turmas para interagir com alunos e tutores.

Com a consolidação destas práticas e a legitimação dos papeis introduzidos na estruturação do curso, os índices de avaliação nas disciplinas seguintes se estabilizaram em níveis elevados - média de $87 \%$ - na percepção dos alunos.

As mudanças implementadas também se refletiram em melhorias nas avaliações dos tutores, conforme pode ser observado na tabela 2 abaixo.

Tabela 2 - índices de aprovação dos tutores de disciplinas

\begin{tabular}{|c|c|c|c|}
\hline \multicolumn{2}{|l|}{ Disciplinas 2006-2 } & \multicolumn{2}{|l|}{ Disciplinas 2007-1 } \\
\hline EAD & $73 \%$ & OSM & $76 \%$ \\
\hline TGA & $71 \%$ & Processo Decisório & $73 \%$ \\
\hline Sociologia & $70 \%$ & Matemática & $73 \%$ \\
\hline Ciência Política & $72 \%$ & Filosofia & $73 \%$ \\
\hline \multicolumn{2}{|l|}{ Disciplinas 2007-2 } & \multicolumn{2}{|l|}{ Disciplinas 2008-1 } \\
\hline Direito Administrativo & $78 \%$ & Sist. De informação & $77 \%$ \\
\hline Contabilidade & $63 \%$ & Matemática Financeira & $69 \%$ \\
\hline Economia & $73 \%$ & Administração Pública & $77 \%$ \\
\hline \multirow{2}{*}{ Psicologia } & \multirow{2}{*}{$87 \%$} & Estatística & $64 \%$ \\
\hline & & Antropologia & $98 \%$ \\
\hline \multicolumn{2}{|l|}{ Disciplinas 2008-2 } & \multicolumn{2}{|l|}{ Disciplinas 2009-1 } \\
\hline Resp. social corporativa & $95 \%$ & Gestão de pessoas & $90 \%$ \\
\hline Adm. Fin. e orçamentária & $84 \%$ & Marketing & $86 \%$ \\
\hline Economia Micro e macro & $68 \%$ & Gestão Ambiental & $91 \%$ \\
\hline Planejamento & $81 \%$ & Comércio exterior* & $79 \%$ \\
\hline
\end{tabular}

Fonte: base de dados de avaliação das disciplinas do curso de graduação.

Os índices de aprovação da atuação dos tutores de disciplinas refletem o conjunto de ações implementadas para dar suporte a este profissional. A ampliação do escopo do processo de capacitação, incluindo questões pedagógicas sobre EAD e o fato de reunir-se tutores, coordenação de tutoria e professores em um mesmo espaço físico durante a realização dos chats, e toda a assistência prestada no processo de avaliação dos alunos são elementos que demonstram a forma como este apoio foi incrementado.

Outro fator que contribuiu para a elevação dos índices de aprovação - média de $86 \%$ - dos tutores nos últimos semestres do curso em foco foi o gerenciamento do banco de tutores. Aqueles que obtinham bons índices de avaliação eram aproveitados em outras disciplinas.

\section{CONSIDERAÇÕES FINAIS}

Os diversos atores que passaram pela graduação em Administração a distância da EA/UFRGS ajudaram a criar, testar e melhorar as práticas administrativas e pedagógicas. Coordenadores, professores e tutores estabeleceram rotinas que, em alguma medida, contribuíram para elevar os índices de satisfação dos alunos. 
No entanto, a introdução de papeis de tutores-facilitadores, coordenadores de tutoria e assistentes de avaliação na estrutura dos cursos, a partir do ano de 2008, proporcionaram maior eficácia ao processo de planejamento e execução dos planos de ensino das disciplinas. Esses aspectos foram mais claramente percebidos pelos alunos e se refletiram positivamente nas avaliações realizadas ao final de cada disciplina.

Ressalta-se, no entanto, que as mudanças ocorreram muito mais em um processo de tentativa e erro, do que fruto de ações coordenadas e planejadas previamente pelos membros das equipes de EAD. Tal fato indica que ainda há muito a avançar em termos de gestão administrativa e pedagógica nos cursos de EAD da EA/UFRGS, bem como em toda a Universidade.

\section{REFERÊNCIAS BIBLIOGRÁFICAS}

KLERING, L. R. \& SCHRÖEDER, C. S. Desenvolvimento de um Ambiente Virtual de Aprendizagem à luz do Enfoque Sistêmico. In: Tecnologias de Administração e Contabilidade, Curitiba, v.1, n. 2, art 1, pp. 42-54, Jul/Dez 2011.

SCHRÖEDER, C. da S. Educação a distância e mudança organizacional na Escola de Administração da UFRGS: uma teoria substantiva. 2009. 252 f. Tese (Doutorado em Administração)-Programa de Pós-Graduação em Administração, Universidade Federal do Rio Grande dos Sul, Porto Alegre.

UFRGS. Avaliação das disciplinas do curso de graduação em Administração modalidade a distância. Disponível em: <http://www.eavirtual.ea.ufrgs.br >. Acesso em: 01 mai. 2010.

UFRGS. Projeto Pedagógico: curso de graduação em Administração - modalidade a distância. 2006. Porto Alegre: UFRGS, Escola de Administração, Comissão de Graduação, Coordenação Graduação a Distância, Março, 2006. 